Working Paper/Document de travail 2011-20

\title{
A Stochastic Volatility Model with Conditional Skewness
}

by Bruno Feunou and Roméo Tédongap 
Bank of Canada Working Paper 2011-20

October 2011

\title{
A Stochastic Volatility Model with Conditional Skewness
}

by

\author{
Bruno Feunou ${ }^{1}$ and Roméo Tédongap ${ }^{2}$ \\ 1Financial Markets Department \\ Bank of Canada \\ Ottawa, Ontario, Canada K1A 0G9 \\ feun@bankofcanada.ca \\ ${ }^{2}$ Department of Finance \\ Stockholm School of Economics \\ Stockholm, Sweden \\ Romeo.Tedongap@hhs.se
}

Bank of Canada working papers are theoretical or empirical works-in-progress on subjects in economics and finance. The views expressed in this paper are those of the authors.

No responsibility for them should be attributed to the Bank of Canada. 


\section{Acknowledgements}

An earlier version of this paper was circulated and presented at various seminars and conferences under the title “Affine Stochastic Skewness.” We thank Nour Meddahi, Glen Keenleyside, Scott Hendry, seminar participants at the Duke University Financial Econometrics Lunch Group, participants at the Conference of the Society for Computational Economics (Montréal, June 2007), the Forecasting in Rio Conference at the Graduate School of Economics (Rio de Janeiro, July 2008), and the Summer Econometric Society Meetings (Boston University, June 2009). 


\begin{abstract}
We develop a discrete-time affine stochastic volatility model with time-varying conditional skewness (SVS). Importantly, we disentangle the dynamics of conditional volatility and conditional skewness in a coherent way. Our approach allows current asset returns to be asymmetric conditional on current factors and past information, what we term contemporaneous asymmetry. Conditional skewness is an explicit combination of the conditional leverage effect and contemporaneous asymmetry. We derive analytical formulas for various return moments that are used for generalized method of moments estimation. Applying our approach to S\&P500 index daily returns and option data, we show that one- and two-factor SVS models provide a better fit for both the historical and the risk-neutral distribution of returns, compared to existing affine generalized autoregressive conditional heteroskedasticity (GARCH) models. Our results are not due to an overparameterization of the model: the one-factor SVS models have the same number of parameters as their one-factor GARCH competitors.
\end{abstract}

JEL classification: C1, C5, G1, G12

Bank classification: Econometric and statistical methods; Asset pricing

\title{
Résumé
}

Les auteurs élaborent un modèle discret affine à volatilité stochastique et asymétrie conditionnelle variable (modèle SVS). Leur approche a ceci d'intéressant qu'elle dissocie de façon cohérente la dynamique de la volatilité conditionnelle de celle de l'asymétrie conditionnelle. Elle permet une distribution asymétrique des rendements courants des actifs conditionnellement aux facteurs du moment et à l'information passée, asymétrie que les auteurs qualifient de «contemporaine». Dans leur modèle, l'asymétrie conditionnelle découle de la combinaison explicite de l'effet de levier conditionnel et de l'asymétrie contemporaine. Les auteurs établissent les formules analytiques pour divers moments des rendements utiles pour l'estimation par la méthode des moments généralisés. En se servant de données relatives aux rendements journaliers de l'indice S\&P 500 et aux options connexes, ils montrent que les modèles SVS à un et à deux facteurs décrivent mieux la distribution des rendements (tant historique que risque neutre) que les modèles GARCH affines existants. Leurs résultats ne tiennent pas à un surparamétrage, puisque les modèles SVS unifactoriels comptent le même nombre de paramètres que les modèles GARCH unifactoriels.

Classification JEL : C1, C5, G1, G12

Classification de la Banque : Méthodes économétriques et statistiques; Évaluation des actifs 


\section{Introduction}

The option-pricing literature holds that generalized autoregressive conditional heteroskedasticity (GARCH) and stochastic volatility (SV) models significantly outperform the Black-Scholes model. However, SV models have traditionally been examined in continuous time and the literature has paid less attention to discrete-time SV option valuation models. This is due to the limitations of existing discrete-time SV models in capturing the characteristics of asset returns that are essential to improve their fit of option data. In particular, these models commonly assume that the conditional distribution of returns is symmetric, violate the positivity of the volatility process or do not allow for

leverage effects. This paper contributes to the literature by examining the implications of allowing conditional asymmetries in discrete-time SV models.

The paper proposes and tests a parsimonious discrete-time affine model with stochastic volatility and conditional skewness. Our focus on the affine class of financial time-series volatility models is motivated by their tractability in empirical applications. In option pricing, for example, European options admit closed-form prices. To the best of our knowledge, there is no discrete-time SV model delivering a closed-form option price that has been empirically tested using option data, in contrast to tests performed in several GARCH models. Heston and Nandi (2000) and Christoffersen et al. (2006) describe examples of one-factor GARCH models that belong to the discrete-time affine class, and feature the conditional leverage effect (both papers) and conditional skewness (only the latter paper) in single-period returns. Christoffersen et al. (2008) provide a two-factor generalization of Heston and Nandi's (2000) model to long- and short-run volatility components. The model features only the leverage effect but not conditional skewness in single-period returns. We compare the performance of the new model to these benchmark GARCH models along several dimensions.

As pointed out by Christoffersen et al. (2006), conditionally nonsymmetric return innovations are critically important, since in option pricing, for example, heteroskedasticity and the leverage effect alone do not suffice to explain the option smirk. However, skewness in their inverse Gaussian GARCH model is still deterministically related to volatility and both undergo the same return shocks, while our proposed model features stochastic volatility. Existing GARCH and SV models also characterize the relation between returns and volatility only through their contemporaneous 
covariance (the so-called leverage effect). In contrast, our modeling approach characterizes the entire distribution of returns conditional upon the volatility factors. We refer to the asymmetry of that distribution as contemporaneous asymmetry, which adds up to the leverage effect to determine conditional skewness.

We show that, in the case of affine models, all unconditional moments of observable returns can be derived analytically. We develop and implement an algorithm for computing these unconditional moments in a general discrete-time affine model that nests our proposed model and all existing affine GARCH models. Jiang and Knight (2002) provide similar results in an alternative way for continuous-time affine processes. They derive the unconditional joint characteristic function of the diffusion vector process in closed form. In discrete time, this can be done only through calculation of unconditional moments, and the issue has not been addressed so far in the literature. Analytical formulas help in assessing the direct impact of model parameters on critical unconditional moments. In particular, this can be useful for calibration exercises where model parameters are estimated to directly match relevant sample moments from the data.

Armed with these unconditional moments, we propose a generalized method of moments (GMM)based estimation of affine GARCH and SV models based on exact moment conditions. Interestingly, the sample variance-covariance matrix of the vector of moments is nonparametric, thus allowing for efficient GMM in one step. This approach is faster and computationally more efficient than alternative estimation methods (see Jacquier et al. 1994; Andersen et al. 1999; Danielsson 1994). Moreover, the minimum distance between model-implied and actual sample return moments appears as a natural metric for comparing different model fits.

Applying this GMM procedure to fit the historical dynamics of observed returns from January 1962 to December 2010, we find that the SVS model characterizes S\&P500 returns well. In addition to the sample mean, variance, skewness and kurtosis of returns, the models are estimated to match the sample autocorrelations of squared returns up to a six-month lag, and the correlations between returns and future squared returns up to a two-month lag. The persistence and the size of these correlations at longer lags cannot be matched by single-factor models. We find that the two-factor models provide the best fit of these moments and, among them, the two-factor SVS model does better than the two-factor GARCH model. 
Our results point out the benefit of allowing for conditional skewness in returns, since the onefactor SVS model with contemporaneous normality fits better than the GARCH model of Heston and Nandi (2000), although both models share the same number of parameters. Our results also show that the SVS model with contemporaneous normality is more parsimonious than the inverse Gaussian GARCH model of Christoffersen et al. (2006), which has one more parameter and nests the GARCH model of Heston and Nandi (2000). In fact, the SVS model with contemporaneous normality and the inverse Gaussian GARCH model have an equal fit of the historical returns distribution.

Fitting the risk-neutral dynamics using S\&P500 option data, we find that explicitly allowing for contemporaneous asymmetry in the one-factor SVS model leads to substantial gains in option pricing, compared to benchmark one-factor GARCH models. We compare models using the option implied-volatility root-mean-square error (IVRMSE). The one-factor SVS model with contemporaneous asymmetry outperforms the two benchmark one-factor GARCH models in the overall fit of option data and across all option categories as well. The IVRMSE of the SVS model is about $23.26 \%$ and $19.85 \%$ below that of the GARCH models. The two-factor models show the best fit of option data overall and across all categories, and they have a comparable fit overall. The two-factor SVS model has an overall IVRMSE of $2.98 \%$, compared to $3.00 \%$ for the two-factor GARCH model.

The rest of the paper is organized as follows. Section 2 discusses existing discrete-time affine GARCH and SV models and their limitations. Section 3 introduces our discrete-time SVS model and discusses the new features relative to existing models. Section 4 estimates univariate and bivariate SVS and GARCH models on S\&P500 index daily returns and provides comparisons and diagnostics. Section 5 estimates univariate and bivariate SVS models, together with competitive GARCH models, using S\&P500 index daily option data, and provides comparisons and diagnostics. Section 6 concludes. An external appendix containing additional materials and proofs is available from the authors' webpages. 


\section{Discrete-Time Affine Models: An Overview}

A discrete-time affine latent-factor model of returns with time-varying conditional moments may be characterized by its conditional log moment-generating function:

$$
\Psi_{t}(x, y ; \theta)=\ln E_{t}\left[\exp \left(x r_{t+1}+y^{\top} l_{t+1}\right)\right]=A(x, y ; \theta)+B(x, y ; \theta)^{\top} l_{t},
$$

where $E_{t}[\cdot] \equiv E\left[\cdot \mid I_{t}\right]$ denotes the expectation conditional on a well-specified information set $I_{t}$, $r_{t}$ is the observable returns, $l_{t}=\left(l_{1 t}, . ., l_{K t}\right)^{\top}$ is the vector of latent factors and $\theta$ is the vector of parameters. Note that the conditional moment-generating function is exponentially linear in the latent variable $l_{t}$ only. Bates (2006) refers to such a process as semi-affine. In what follows, the parameter $\theta$ is withdrawn from functions $A$ and $B$ for expositional purposes. In this section, we discuss discrete-time affine GARCH and SV models and their limitations, which we want to overcome by introducing a new discrete-time affine SV model featuring conditional skewness.

The following SV models are discrete-time semi-affine univariate latent-factor models of returns considered in several empirical studies. The dynamics of returns is given by

$$
r_{t+1}=\mu_{r}-\lambda_{h} \mu_{h}+\lambda_{h} h_{t}+\sqrt{h_{t}} u_{t+1},
$$

where the volatility process satisfies one of the following:

$$
\begin{aligned}
& h_{t+1}=\left(1-\phi_{h}\right) \mu_{h}-\alpha_{h}+\left(\phi_{h}-\alpha_{h} \beta_{h}^{2}\right) h_{t}+\alpha_{h}\left(\varepsilon_{t+1}-\beta_{h} \sqrt{h_{t}}\right)^{2}, \\
& h_{t+1}=\left(1-\phi_{h}\right) \mu_{h}+\phi_{h} h_{t}+\sigma_{h} \varepsilon_{t+1}, \\
& h_{t+1}=\left(1-\phi_{h}\right) \mu_{h}+\phi_{h} h_{t}+\sigma_{h} \sqrt{h_{t}} \varepsilon_{t+1},
\end{aligned}
$$

and where $u_{t+1}$ and $\varepsilon_{t+1}$ are two i.i.d. standard normal shocks. The parameter vector $\theta$ is $\left(\mu_{r}, \lambda_{h}, \mu_{h}, \phi_{h}, \alpha_{h}, \beta_{h}, \rho_{r h}\right)^{\top}$ with volatility dynamics $(3)$, whereas it is $\left(\mu_{r}, \lambda_{h}, \mu_{h}, \phi_{h}, \sigma_{h}\right)^{\top}$ with au-

toregressive Gaussian volatility (4) and finally $\left(\mu_{r}, \lambda_{h}, \mu_{h}, \phi_{h}, \sigma_{h}, \rho_{r h}\right)^{\top}$ with square-root volatility (5), where $\rho_{r h}$ denotes the conditional correlation between the shocks $u_{t+1}$ and $\varepsilon_{t+1}$. The special case $\rho_{r h}=1$ in the volatility dynamics (3) corresponds to Heston and Nandi's (2000) GARCH 
model, which henceforth we refer to as HN.

Note that volatility processes (4) and (5) are not well defined, since $h_{t}$ can take negative values. This can also arise with process (3) unless the parameters satisfy a couple of constraints. In simulations, for example, one should be careful when using a reflecting barrier at a small positive number to ensure the positivity of simulated volatility samples. Besides, if the volatility shock $\varepsilon_{t+1}$ in (4) is allowed to be correlated to the return shock $u_{t+1}$ in (2), then the model loses its affine property. Also notice that the conditional skewness of returns in these models is zero.

Christoffersen et al. (2006) propose an affine GARCH model that allows conditional skewness in returns, specified by

$$
\begin{aligned}
r_{t+1} & =\gamma_{h}+\nu_{h} h_{t}+\eta_{h} y_{t+1}, \\
h_{t+1} & =w_{h}+b_{h} h_{t}+c_{h} y_{t+1}+a_{h} \frac{h_{t}^{2}}{y_{t+1}},
\end{aligned}
$$

where, given the available information at time $t, y_{t+1}$ has an inverse Gaussian distribution with the degree-of-freedom parameter $h_{t} / \eta_{h}^{2}$. Alternatively, $y_{t+1}$ may be written as

$$
y_{t+1}=\frac{h_{t}}{\eta_{h}^{2}}+\frac{\sqrt{h_{t}}}{\eta_{h}} z_{t+1}
$$

where $z_{t+1}$ follows a standardized inverse Gaussian distribution with parameter $s_{t}=3 \eta_{h} / \sqrt{h_{t}}$. The standardized inverse Gaussian distribution is introduced in Section 3.1.1. Interestingly, Christoffersen et al. (2006) provide a reparameterization of their model so that HN appears to be a limit as $\eta_{h}$ approaches zero:

$$
\begin{aligned}
& a_{h}=\frac{\alpha_{h}}{\eta_{h}^{4}}, \quad b_{h}=\phi_{h}-\frac{\alpha_{h}}{\eta_{h}^{2}}-\frac{\alpha_{h}-2 \alpha_{h} \eta_{h} \beta_{h}}{\eta_{h}^{2}}, \quad c_{h}=\alpha_{h}-2 \alpha_{h} \eta_{h} \beta_{h}, \\
& w_{h}=\left(1-\phi_{h}\right) \mu_{h}-\alpha_{h}, \quad \gamma_{h}=\mu_{r}-\lambda_{h} \mu_{h}, \quad \nu_{h}=\lambda_{h}-\frac{1}{\eta_{h}} .
\end{aligned}
$$

Henceforth we refer to this specification as CHJ.

While CHJ allows for both the leverage effect and conditional skewness, it does not separate the volatility of volatility from the leverage effect on the one hand, and conditional skewness from volatility on the other hand. In particular, conditional skewness and volatility are related by 
$s_{t}=3 \eta_{h} / \sqrt{h_{t}}$. In consequence, the sign of conditional skewness is constant over time and equal to the sign of the parameter $\eta_{h}$. This contrasts with the empirical evidence in Harvey and Siddique (1999) that conditional skewness changes sign over time. Feunou et al.'s (2011) findings also suggest that, although conditional skewness is centered around a negative value, return skewness may take positive values.

Christoffersen et al. (2008) introduce a two-factor generalization of HN to long- and short-run volatility components, which henceforth we refer to as CJOW. In addition to the dynamics of return (2), the volatility dynamics may be written as follows:

$$
h_{t}=h_{1, t}+h_{2, t} \quad \text { where }\left\{\begin{array}{l}
h_{1, t+1}=\mu_{1 h}+\phi_{1 h} h_{1, t}+\alpha_{1 h} u_{t+1}^{2}-2 \alpha_{1 h} \beta_{1 h} \sqrt{h_{t}} u_{t+1} \\
h_{2, t+1}=\mu_{2 h}+\phi_{2 h} h_{2, t}+\alpha_{2 h} u_{t+1}^{2}-2 \alpha_{2 h} \beta_{2 h} \sqrt{h_{t}} u_{t+1},
\end{array}\right.
$$

with $\mu_{1 h}=0$, since only the sum $\mu_{1 h}+\mu_{2 h}$ is identifiable.

Liesenfeld and Jung (2000) introduce SV models with conditional heavy tails, but their model is non-affine. However, SV models with conditional asymmetry have received less attention so far. In this paper, we aim to combine in a coherent way both the affine property and the ability of an SV model to fit critical moments of the data (mean, variance, skewness, kurtosis, multipleday autocorrelation of squared returns and cross-correlation between returns are future squared returns). In the next section, we develop an affine multivariate latent-factor model of returns such that both conditional variance $h_{t}$ and conditional skewness $s_{t}$ are stochastic. We refer to such a model as SVS. The proposed model is parsimonious and solves for the limitations of existing models. Later in Sections 4 and 5, we use S\&P500 index returns and option data to examine the relative performance of the one- and two-factor SVS to the GARCH alternatives (HN, CHJ and CJOW).

\section{Building an SV Model with Conditional Skewness}

\subsection{The Model Structure}

For each variable in what follows, the time subscript denotes the date from which the value of the variable is observed by the economic agent; to simplify notations, the usual scalar operators will 
also apply to vectors element-by-element. The joint distribution of returns $r_{t+1}$ and latent factors $\sigma_{t+1}^{2}$ conditional on previous information denoted $I_{t}$ and containing previous realizations of returns $\underline{r_{t}}=\left\{r_{t}, r_{t-1}, \ldots\right\}$ and latent factors $\underline{\sigma_{t}^{2}}=\left\{\sigma_{t}^{2}, \sigma_{t-1}^{2}, \ldots\right\}$ may be decomposed as follows:

$$
f\left(r_{t+1}, \sigma_{t+1}^{2} \mid I_{t}\right) \equiv f_{c}\left(r_{t+1} \mid \sigma_{t+1}^{2}, I_{t}\right) \times f_{m}\left(\sigma_{t+1}^{2} \mid I_{t}\right)
$$

Based on this, our modeling strategy consists of specifying, in a first step, the distribution of returns conditional on factors and previous information, and, in a second step, the dynamics of the factors. The first step will be characterized by inverse Gaussian shocks, and the second step will follow a multivariate autoregressive gamma process.

\subsubsection{Standardized Inverse Gaussian Shocks}

The dynamics of returns in our model is built upon shocks drawn from a standardized inverse Gaussian distribution. The inverse Gaussian process has been investigated by Jensen and Lunde (2001), Forsberg and Bollerslev (2002), and Christoffersen et al. (2006). See also the excellent overview of related processes in Barndorff-Nielsen and Shephard (2001).

The log moment-generating function of a discrete random variable that follows a standardized inverse Gaussian distribution of parameter $s$, denoted $S I G(s)$, is given by

$$
\psi(u ; s)=\ln E[\exp (u X)]=-3 s^{-1} u+9 s^{-2}\left(1-\sqrt{1-\frac{2}{3} s u}\right) .
$$

For such a random variable, one has $E[X]=0, E\left[X^{2}\right]=1$ and $E\left[X^{3}\right]=s$, meaning that $s$ is the skewness of $X$. In addition to the fact that the $S I G$ distribution is directly parameterized by its skewness, the limiting distribution when the skewness $s$ tends to zero is the standard normal distribution, that is $S I G(0) \equiv \mathcal{N}(0,1)$. This particularity makes the $S I G$ an ideal building block for studying departures from normality.

\subsubsection{Autoregressive Gamma Latent Factors}

The conditional distribution of returns is further characterized by $K$ latent factors, the components

of the $K$-dimensional vector process $\sigma_{t+1}^{2}$. We assume that $\sigma_{t+1}^{2}$ is a multivariate autoregressive 
gamma process with mutually independent components. We use this process to guarantee the positivity of the volatility factors so that volatility itself is well defined. Its cumulant-generating function, conditional on $I_{t}$, is given by

$$
\begin{aligned}
& \Psi_{t}^{\sigma}(y) \equiv \ln E\left[\exp \left(y^{\top} \sigma_{t+1}^{2}\right) \mid I_{t}\right]=\sum_{i=1}^{K} f_{i}\left(y_{i}\right)+\sum_{i=1}^{K} g_{i}\left(y_{i}\right) \sigma_{i, t}^{2}, \\
& f_{i}\left(y_{i}\right)=-\nu_{i} \ln \left(1-\alpha_{i} y_{i}\right) \text { and } g_{i}\left(y_{i}\right)=\frac{\phi_{i} y_{i}}{1-\alpha_{i} y_{i}}
\end{aligned}
$$

Each factor $\sigma_{i, t}^{2}$ is a univariate autoregressive gamma process, which is an $\operatorname{AR}(1)$ process with persistence parameter $\phi_{i}$. The parameters $\nu_{i}$ and $\alpha_{i}$ are related to persistence, unconditional mean $\mu_{i}$ and unconditional variance $\omega_{i}$ as $\nu_{i}=\mu_{i}^{2} / \omega_{i}$ and $\alpha_{i}=\left(1-\phi_{i}\right) \omega_{i} / \mu_{i}$. A more in-depth treatment of the univariate autoregressive gamma process can be found in Gourieroux and Jasiak (2006) and Darolles et al. (2006). Their analysis is extended to the multivariate case and applied to the term structure of interest rates modeling by Le et al. (2010). The autoregressive gamma process also represents the discrete-time counterpart to the continuous-time square-root process that has previously been examined in the SV literature (see, for example, Singleton 2006, p. 110).

We denote by $m_{t}^{\sigma}, v_{t}^{\sigma}$ and $\xi_{t}^{\sigma}$ the $K$-dimensional vectors of conditional means, variances, and third moments of the individual factors, respectively. Their $i$ th component is given by

$$
\begin{aligned}
& m_{i, t}^{\sigma}=\left(1-\phi_{i}\right) \mu_{i}+\phi_{i} \sigma_{i, t}^{2} \text { and } v_{i, t}^{\sigma}=\left(1-\phi_{i}\right)^{2} \omega_{i}+\frac{2\left(1-\phi_{i}\right) \phi_{i} \omega_{i}}{\mu_{i}} \sigma_{i, t}^{2}, \\
& \xi_{i, t}^{\sigma}=\frac{2\left(1-\phi_{i}\right)^{3} \omega_{i}^{2}}{\mu_{i}}+\frac{6\left(1-\phi_{i}\right)^{2} \phi_{i} \omega_{i}^{2}}{\mu_{i}^{2}} \sigma_{i, t}^{2} .
\end{aligned}
$$

The $\operatorname{AR}(1)$ process $\sigma_{i, t}^{2}$ thus has the formal representation

$$
\sigma_{i, t+1}^{2}=\left(1-\phi_{i}\right) \mu_{i}+\phi_{i} \sigma_{i, t}^{2}+\sqrt{v_{i, t}^{\sigma}} z_{i, t+1}
$$

where $v_{i, t}^{\sigma}$ is given in equation (14) and $z_{i, t+1}$ is an error with mean zero and unit variance and skewness $\xi_{i, t}^{\sigma}\left(v_{i, t}^{\sigma}\right)^{-3 / 2}$. The conditional density function of an autoregressive gamma process is obtained as a convolution of the standard gamma and Poisson distributions. A discussion and a formal expression of that density can be found in Singleton (2006, p. 109). 


\subsubsection{The Dynamics of Returns}

Formally, we assume that logarithmic returns have the following dynamics:

$$
r_{t+1}=\ln \frac{P_{t+1}}{P_{t}}=\mu_{t}^{r}+u_{t+1}^{r}
$$

where $P$ is the price of the asset, $\mu_{t}^{r} \equiv E_{t}\left[r_{t+1} \mid I_{t}\right]$ denotes the expected (or conditional mean of) returns, which we assume are given by

$$
\mu_{t}^{r}=\lambda_{0}+\lambda^{\top} \sigma_{t}^{2}
$$

and $u_{t+1}^{r} \equiv r_{t+1}-E_{t}\left[r_{t+1} \mid I_{t}\right]$ represents the unexpected (or innovation of) returns, which we assume are given by

$$
u_{t+1}^{r}=\beta^{\top}\left(\sigma_{t+1}^{2}-m_{t}^{\sigma}\right)+\sigma_{t+1}^{\top} u_{t+1}
$$

Our modeling strategy thus decomposes unexpected returns into two parts: a contribution due to factor innovations and another due to shocks that are orthogonal to factor innovations. We assume that the $i$ th component of this $K$-dimensional vector of shocks $u_{t+1}$ has a standardized inverse Gaussian distribution, conditional on factors and past information,

$$
u_{i, t+1} \mid\left(\sigma_{t+1}^{2}, I_{t}\right) \sim S I G\left(\eta_{i} \sigma_{i, t+1}^{-1}\right)
$$

and that the $K$ return shocks are mutually independent conditionally on $\left(\sigma_{t+1}^{2}, I_{t}\right)$. If $\eta_{i}=0$, then $u_{i, t+1}$ is a standard normal shock.

Under these assumptions, we have

$$
\ln E\left[\exp \left(x r_{t+1}\right) \mid \sigma_{t+1}^{2}, I_{t}\right]=\left(\mu_{t}^{r}-\beta^{\top} m_{t}^{\sigma}\right) x+\sum_{i=1}^{K}\left(\beta_{i} x+\psi\left(x ; \eta_{i}\right)\right) \sigma_{i, t+1}^{2}
$$

where the function $\psi(\cdot, s)$ is the cumulant-generating function of the standardized inverse Gaussian distribution with skewness $s$ as defined in equation (12). In total, the model has $1+6 K$ parameters grouped in the vector $\theta=\left(\lambda_{0}, \lambda^{\top}, \beta^{\top}, \eta^{\top}, \mu^{\top}, \phi^{\top}, \omega^{\top}\right)^{\top}$. The scalar $\lambda_{0}$ is the drift coefficient in conditional expected returns. All vector parameters in $\theta$ are $K$-dimensional. Namely, the vector $\lambda$ 
contains loadings of expected returns on the $K$ factors, the vector $\beta$ contains loadings of returns on the $K$ factor innovations, the vector $\eta$ contains skewness coefficients of the $K$ standardized inverse Gaussian shocks, and the vectors $\mu, \phi$ and $\omega$ contain unconditional means, persistence and variances of the $K$ factors, respectively.

Although, for the purpose of this paper, we limit ourselves to a single-return setting, the model admits a straightforward generalization to multiple returns. Also, we further limit our empirical application in this paper to one and two factors. Since the empirical evidence regarding the timevarying conditional mean is weak from historical index daily returns data, we will restrict ourselves in the estimation section to $\lambda=0$ and will pick $\lambda_{0}$ to match the sample unconditional mean of returns, leaving us with $5 K$ critical parameters from which further interesting restrictions can be considered.

\subsection{Volatility, Conditional Skewness and the Leverage Effect}

In the previous subsection, we did not model conditional volatility and skewness or other higher moments of returns directly. Instead, we related returns to stochastic linearly independent positive factors. In this section, we derive useful properties of the model and discuss its novel features in relation to the literature. In particular, we show that, in addition to stochastic volatility and the leverage effect, the model generates conditional skewness. This nonzero and stochastic conditional skewness, coupled with the ability of the model to generalize to multiple returns and multiple factors, constitutes the main significant difference from previous affine SV models in discrete time.

The conditional variance, $h_{t}$, and the conditional skewness, $s_{t}$, of returns, $r_{t+1}$, may be expressed as follows:

$$
\begin{aligned}
& h_{t} \equiv E_{t}\left[\left(r_{t+1}-\mu_{t}^{r}\right)^{2} \mid I_{t}\right]=\iota^{\top} m_{t}^{\sigma}+\left(\beta^{2}\right)^{\top} v_{t}^{\sigma}=\sum_{i=1}^{K} h_{i, t}, \\
& s_{t} h_{t}^{3 / 2} \equiv E_{t}\left[\left(r_{t+1}-\mu_{t}^{r}\right)^{3} \mid I_{t}\right]=\eta^{\top} m_{t}^{\sigma}+3 \beta^{\top} v_{t}^{\sigma}+\left(\beta^{3}\right)^{\top} \xi_{t}^{\sigma}=\sum_{i=1}^{K} \varrho_{i, t},
\end{aligned}
$$

with

$$
h_{i, t}=c_{0 i, h}+c_{i, h} \sigma_{i, t}^{2} \text { and } \varrho_{i, t}=c_{0 i, s}+c_{i, s} \sigma_{i, t}^{2}
$$


where $\iota$ is the $K$-dimensional vector of ones, and the coefficients $c_{i, h}$ and $c_{i, s}$ depend on model parameters $\theta$. These coefficients are explicitly given by

$$
\begin{aligned}
& c_{0 i, h}=\left(1-\phi_{i}\right)\left(\mu_{i}+\left(1-\phi_{i}\right) \omega_{i} \beta_{i}^{2}\right) \text { and } c_{i, h}=\left(1+\frac{2\left(1-\phi_{i}\right) \omega_{i} \beta_{i}^{2}}{\mu_{i}}\right) \phi_{i}, \\
& c_{0 i, s}=\left(1-\phi_{i}\right)\left(\eta_{i} \mu_{i}+3\left(1-\phi_{i}\right) \omega_{i} \beta_{i}+\frac{2\left(1-\phi_{i}\right)^{2} \omega_{i}^{2} \beta_{i}^{3}}{\mu_{i}}\right), \\
& c_{i, s}=\left(\eta_{i}+\frac{6\left(1-\phi_{i}\right) \omega_{i} \beta_{i}}{\mu_{i}}+\frac{6\left(1-\phi_{i}\right)^{2} \omega_{i}^{2} \beta_{i}^{3}}{\mu_{i}^{2}}\right) \phi_{i} .
\end{aligned}
$$

Conditional on $I_{t}$, covariance between returns $r_{t+1}$ and volatility $h_{t+1}$ (the leverage effect) may be expressed as:

$$
\operatorname{Cov}\left(r_{t+1}, h_{t+1} \mid I_{t}\right)=\left(\beta c_{h}\right)^{\top} v_{t}^{\sigma}=\sum_{i=1}^{K} \vartheta_{i, t} \text { with } \vartheta_{i, t}=c_{0 i, r h}+c_{i, r h} \sigma_{i, t}^{2},
$$

where $c_{h}=\left(c_{1, h}, c_{2, h}, \ldots, c_{K, h}\right)^{\top}$ and the coefficients $c_{i, r h}$ are explicitly given by

$$
\begin{aligned}
& c_{0 i, r h}=\left(1+\frac{2\left(1-\phi_{i}\right) \omega_{i} \beta_{i}^{2}}{\mu_{i}}\right)\left(1-\phi_{i}\right)^{2} \phi_{i} \omega_{i} \beta_{i}, \\
& c_{i, r h}=2\left(1+\frac{2\left(1-\phi_{i}\right) \omega_{i} \beta_{i}^{2}}{\mu_{i}}\right) \frac{\left(1-\phi_{i}\right) \phi_{i}^{2} \omega_{i} \beta_{i}}{\mu_{i}} .
\end{aligned}
$$

It is not surprising that the parameter $\beta$ alone governs the conditional leverage effect, since it represents the slope of the linear projection of returns on factor innovations. In particular, for the one-factor SVS model to generate a negative correlation between spot returns and variance as postulated by Black (1976) and documented by Christie (1982) and others, the parameter $\beta_{1}$ should be negative.

In our SVS model, contemporaneous asymmetry $\eta$, alone, does not characterize conditional skewness, as shown in equation (22). The parameter $\beta$, which alone characterizes the leverage effect, also plays a central role in generating conditional asymmetry in returns, even when $\eta=0$. In contrast to SV models discussed in Section 2, where the leverage effect generates skewness only in the multiple-period conditional distribution of returns, in our setting it invokes skewness in the single-period conditional distribution as well. 
To better understand the flexibility of the SVS model in generating conditional skewness, we consider the one-factor SVS without loss of generality. The left-hand side of the last equality in equation (22) shows that conditional skewness is the sum of three terms. The first term has the sign of $\eta_{1}$ and the last two terms have the same sign of $\beta_{1}$. A negative $\beta_{1}$ is necessary to generate the well-documented leverage effect. In that case, the last two terms in (22) are negative. The sign of conditional skewness will then depend on $\eta_{1}$. If $\eta_{1}$ is zero or negative, then conditional skewness is negative over time, as in CHJ. Note that conditional skewness may change sign over time if $\eta_{1}$ is positive and $c_{01, s} c_{1, s}<0$. There are lower and upper positive bounds on $\eta_{1}$ such that this latter condition holds. These bounds are, respectively, $-3\left(1-\phi_{1}\right) \omega_{1} \beta_{1} / \mu_{1}-2\left(1-\phi_{1}\right)^{2} \omega_{1}^{2} \beta_{1}^{3} / \mu_{1}^{2}$ and $-6\left(1-\phi_{1}\right) \omega_{1} \beta_{1} / \mu_{1}-6\left(1-\phi_{1}\right)^{2} \omega_{1}^{2} \beta_{1}^{3} / \mu_{1}^{2}$. This shows that the one-factor SVS model can generate a more realistic time series of conditional skewness compared to CHJ.

We acknowledge that extensions of the basic SV model in continuous time can capture the stylized facts of daily asset prices just as well as the SVS model introduced in this paper. However, the econometrics required for estimating continuous-time processes are demanding, because of the complexity of the resulting filtering and sampling. The advantage of our discrete-time affine approach is not only that it gives an alternative to discrete-time users, but also that discrete-time GARCH and SV models provide straightforward tools to deal with estimation and inference. In the external appendix, we show that although the current SVS model is written in discrete time and is easily applicable to discrete data, it admits interesting continuous-time limits, including the standard SV model of Heston (1993) and an SV model with a jump process with stochastic intensity.

In the next section, we develop an estimation procedure for the one- and two-factor SVS models together with their competitors, HN, CHJ and CJOW. We seek a unified framework where these different models can be estimated and evaluated according to the same criteria, thereby facilitating their empirical comparison. Our proposed framework uses the generalized method of moments to estimate, test and compare the models under consideration. It exploits the affine property of the models to compute analytically model-implied unconditional moments of returns that are further compared to their empirical counterparts. We describe our approach in detail in the next section, and in Section 5 we compare the option-pricing performance of the models. 


\section{SVS vs. GARCH Models: Time-Series Analysis}

\subsection{Analytical Expressions of Unconditional Moments}

Given the joint conditional log moment-generating function (1) of returns and latent variables, the unconditional $\log$ moment-generating function of the latent vector $l_{t}$, denoted by $\Psi_{l}(\cdot)$, satisfies

$$
\Psi_{l}(y)=A_{l}(y)+\Psi_{l}\left(B_{l}(y)\right)
$$

where $A_{l}(y) \equiv A(0, y)$ and $B_{l}(y) \equiv B(0, y)$. Proof of equation (27) can be found in the external appendix. The function $\Psi_{l}(\cdot)$ obtains analytically in some cases, for instance the affine jumpdiffusion processes, as in Jiang and Knight (2002). In a discrete-time setting, it is sufficient to find the derivatives of $\Psi_{l}(y)$ at $y=0$, and this can be done through differentiation of equation (27).

We show that the $n$th unconditional cumulant of the latent vector $l_{t}$ is the $K^{n-1} \times K$ matrix $\kappa_{l}(n) \equiv \kappa_{l}(n)=\mathcal{D}^{n} \Psi_{l}(0)$, where $\mathcal{D}^{n} \Psi_{l}(0)$ is the solution to the equation

$$
\mathcal{D}^{n} \Psi_{l}(0)=\mathcal{D}^{n} A_{l}(0)+\left.\mathcal{D}^{n}\left(\Psi_{l}\left(B_{l}(y)\right)\right)\right|_{y=0},
$$

and depends on $\mathcal{D} \Psi_{l}(0), \mathcal{D}^{2} \Psi_{l}(0), \ldots, \mathcal{D}^{n-1} \Psi_{l}(0), \mathcal{D} B_{l}(0), \mathcal{D}^{2} B_{l}(0), \ldots, \mathcal{D}^{n} B_{l}(0)$, and where the operator $\mathcal{D}$ defines the Jacobian of a matrix function of a matrix variable, as in Magnus and Neudecker (1988, p. 173).

The higher-order derivatives of the composite function in the right-hand side of equation (28) are evaluated through the chain rule given by Faà di Bruno's formula, of which the multivariate version is discussed in detail in Constantine and Savits (1996). In the case of a univariate latent variable $\left(l_{t}\right.$ is scalar), it is easy to solve equation (28) for higher-order cumulants of the latent variable. However, this task is more cumbersome and tedious when $l_{t}$ is a vector. In the latter case, the solution to equation (28) for $n=1$, which is for the first cumulant, is given by

$$
\mathcal{D} \Psi_{l}(0)=\mathcal{D} A_{l}(0)\left[I d_{K}-\mathcal{D} B_{l}(0)\right]^{-1}
$$

where $I d_{K}$ denotes the $K \times K$ identity matrix and $\mathcal{D} B_{l}(0)$ represents the persistence matrix of the 
latent vector $l_{t}$. When $n>1$, which is for the second- and higher-order cumulant, it can be shown that the matrix $\mathcal{D}^{n} \Psi_{l}(0)$ satisfies

$$
\mathcal{D}^{n} \Psi_{l}(0)-\left(\left(\mathcal{D} B_{l}(0)\right)^{\otimes(n-1)}\right)^{\top} \mathcal{D}^{n} \Psi_{l}(0) \mathcal{D} B_{l}(0)=\mathcal{D}^{n} A_{l}(0)+C_{n},
$$

where the matrix $C_{n}$ depends on the matrices $\left\{\mathcal{D}^{j} B_{l}(0)\right\}_{1 \leq j \leq n-1}$ and $\left\{\mathcal{D}^{j} \Psi_{l}(0)\right\}_{2 \leq j \leq n}$ through the multivariate version of Faà di Bruno's formula. For example, the second unconditional cumulant of the latent vector is given by

$$
\mathcal{D}^{2} \Psi_{l}(0)-\mathcal{D} B_{l}(0)^{\top} \mathcal{D}^{2} \Psi_{l}(0) \mathcal{D} B_{l}(0)=\mathcal{D}^{2} A_{l}(0)+\left(I d_{K} \otimes \mathcal{D} \Psi_{l}(0)\right) \mathcal{D}^{2} B_{l}(0)
$$

Equation (30) shows that $\mathcal{D}^{n} \Psi_{l}(0)$ is the solution to a matrix equation of the form $X-\Delta X \Gamma=$ $\Lambda$. The solution to that equation is given by

$$
\operatorname{vec}(X)=\left[I d-\left(\Gamma^{\top} \otimes \Delta\right)\right]^{-1} \operatorname{vec}(\Lambda)
$$

Moments and cross-moments of returns can also be computed analytically, and this can be performed through cross-cumulants of couples $\left(r_{t+1}, r_{t+1+j}\right)^{\top}, j>0$. The unconditional log momentgenerating function of such couples is easily obtained in the case of affine models (see Darolles et al. 2006). It is given by

$$
\Psi_{r, j}(x, z)=A_{r, j}(z)+A\left(x, B_{r, j}(z)\right)+\Psi_{l}\left(B\left(x, B_{r, j}(z)\right)\right)
$$

where the functions $A_{r, j}$ and $B_{r, j}$ satisfy the forward recursions

$$
\begin{aligned}
& A_{r, j}(z)=A_{r, j-1}(z)+A_{l}\left(B_{r, j-1}(z)\right) \\
& B_{r, j}(z)=B_{l}\left(B_{r, j-1}(z)\right)
\end{aligned}
$$

with the initial conditions $A_{r, 1}(z)=A(z, 0)$ and $B_{r, 1}(z)=B(z, 0)$.

Given $n>0$ and $m>0$, the unconditional cross-cumulant of order $(n, m)$ of the observable 
returns $r_{t}$ is the number $\kappa_{r, j}(n, m) \equiv \frac{\partial^{n+m} \Psi_{r, j}}{\partial x^{n} \partial z^{m}}(0,0)$ where $\frac{\partial^{n+m} \Psi_{r, j}}{\partial x^{n} \partial z^{m}}(0,0)$ is the solution to

$$
\frac{\partial^{n+m} \Psi_{r, j}}{\partial x^{n} \partial z^{m}}(0,0)=\left.\frac{\partial^{n+m}}{\partial x^{n} \partial z^{m}}\left(A\left(x, B_{r, j}(z)\right)\right)\right|_{x=0, z=0}+\left.\frac{\partial^{n+m}}{\partial x^{n} \partial z^{m}}\left(\Psi_{l}\left(B\left(x, B_{r, j}(z)\right)\right)\right)\right|_{x=0, z=0}
$$

Equation (35) shows that cumulants of the latent vector $l_{t}$ are essential to compute cumulants and cross-cumulants of returns.

We have just provided analytical formulas for computing return cumulants and cross-cumulants $\kappa_{r, j}(n, m), j>0, n \geq 0, m>0$. This also allows us to compute analytically the corresponding

return moments and cross-moments $\mu_{r, j}(n, m)=E\left[r_{t}^{n} r_{t+j}^{m}\right]$ through the relationship between multivariate moments and cumulants.

\subsection{GMM Procedure}

All the moments previously computed are functions of the parameter vector $\theta$ that governs the joint dynamics of returns and the latent factors. We can then choose $N$ pertinent moments to perform the GMM estimation of the returns model. In this paper, we choose $N$ pertinent moments among all the moments $\mu_{r, j}(n, m)=E\left[r_{t}^{n} r_{t+j}^{m}\right]$ such that $j \geq 1, n \geq 0$ and $m>0$. Since the moments of observed returns implied by a given model can directly be compared to their sample equivalent, our estimation setup evaluates the performance of a given model in replicating well-known stylized facts.

Let $g_{t}(\theta)=\left[r_{t}^{n_{i}} r_{t+j_{i}}^{m_{i}}-\mu_{r, j_{i}}\left(n_{i}, m_{i}\right)\right]_{1 \leq i \leq N}$ denote the $N \times 1$ vector of the chosen moments. We have $E\left[g_{t}(\theta)\right]=0$ and we define the sample counterpart of this moment condition as follows:

$$
\widehat{g}(\theta)=\left(\begin{array}{c}
\widehat{E}\left[r_{t}^{n_{1}} r_{t+j_{1}}^{m_{1}}\right]-\mu_{r, j_{1}}\left(n_{1}, m_{1}\right) \\
\cdots \\
\widehat{E}\left[r_{t}^{n_{N}} r_{t+j_{N}}^{m_{N}}\right]-\mu_{r, j_{N}}\left(n_{N}, m_{N}\right)
\end{array}\right)
$$

Given the $N \times N$ matrix $\widehat{W}$ used to weight the moments, the GMM estimator $\widehat{\theta}$ of the parameter vector is given by

$$
\widehat{\theta}=\arg \min _{\theta} T\left(\widehat{g}(\theta)^{\top} \widehat{W} \widehat{g}(\theta)\right)
$$


where $T$ is the sample size. Interestingly, the heteroskedasticity and autocorrelation (HAC) estimator of the variance-covariance matrix of $g_{t}(\theta)$ is simply that of the variance-covariance matrix of $\left[r_{t}^{n_{i}} r_{t+j_{i}}^{m_{i}}\right]_{1 \leq i \leq N}$, which does not depend on the vector of parameter $\theta$. This is an advantage, since with a nonparametric empirical variance-covariance matrix of moment conditions, the optimal GMM procedure can be implemented in one step. It is also important to note that two different models can be estimated via the same moment conditions and weighting matrix. Only the modelimplied moments $\left[\mu_{r, j_{i}}\left(n_{i}, m_{i}\right)\right]_{1 \leq i \leq N}$ differ from one model to another in this estimation procedure. In this case, the minimum value of the GMM objective function itself is a criterion for comparison of the alternative models, since it represents the distance between the model-implied moments and the actual moments.

We weigh the moments using the inverse of the diagonal of their long-run variance-covariance matrix:

$$
\widehat{W}=\left\{\operatorname{Diag}\left(\widehat{\operatorname{Var}}\left[g_{t}\right]\right)\right\}^{-1}
$$

This matrix is nonparametric and puts more weight on moments with low magnitude. If the number of moments to match is large, as is the case in our estimation in the next section, then inverting the long-run variance-covariance matrix of moments will be numerically unstable. Using the inverse of the diagonal instead of the inverse of the long-run variance-covariance matrix itself allows for numerical stability if the number of moments to match is large, since inverting a diagonal matrix is simply taking the diagonal of the inverse of its diagonal elements. The distance to minimize reduces to

$$
\sum_{i=1}^{N}\left(\frac{\widehat{E}\left[r_{t}^{n_{i}} r_{t+j_{i}}^{m_{i}}\right]-E\left[r_{t}^{n_{i}} r_{t+j_{i}}^{m_{i}}\right]}{\widehat{\sigma}\left[r_{t}^{n_{i}} r_{t+j_{i}}^{m_{i}}\right] / \sqrt{T}}\right)^{2}
$$

where observed moments are denoted with a hat and the model-implied theoretical moment without.

In some cases, this GMM procedure has a numerical advantage compared to the maximumlikelihood estimation even when the likelihood function can be derived. Maximum-likelihood estimation becomes difficult to perform numerically and theoretically, especially when the support of the likelihood function is parameter-dependent. While the appeal of GARCH models relies on the availability of their likelihood function in analytical form, which eases their estimation, the 
support of the likelihood function for CHJ is parameter-dependent. This complicates its estimation by maximum likelihood and, most importantly, its inference. In fact, there exists no general theory in the statistical literature about the distributional properties of the maximum-likelihood estimator when the support of the likelihood function is parameter-dependent.

On the other hand, the maximum-likelihood estimation of semi-affine latent variable models of Bates (2006) and the quasi-maximum-likelihood estimation based on the Kalman recursion have the downside that critical unconditional higher moments (skewness and kurtosis) of returns can be poorly estimated due to the second-order approximation of the distribution of the latent variable conditional on observable returns. Moreover, in single-stage estimation and filtering methods such as the unscented Kalman filter and Bates's (2006) algorithm, approximations affect both parameter and state estimations.

Conversely, our GMM procedure matches critical higher moments exactly and requires no approximation for parameter estimation. Given GMM estimates of model parameters, Bates's (2006) procedure, or any other filtering procedure, such as the unscented Kalman filter, can be followed for the state estimation. In this sense, approximations required by these techniques affect only state estimation.

\subsection{Data and Parameter Estimation}

Using daily returns on the S\&P500 equal-weighted index from January 2, 1962 to December 31, 2010, we estimate the 5-parameter unconstrained one-factor SVS model, the 4-parameter onefactor SVS model with the constraint $\eta_{1}=0$ (contemporaneous normality), and the 10-parameter unconstrained two-factor SVS model, which we respectively denote SVS1FU, SVS1FC and SVS2FU. We also estimate their GARCH competitors, the one-factor models CHJ with five parameters and HN with four parameters, and the two-factor model CJOW with seven parameters.

To perform the GMM procedure, we need to decide which moments to consider. The top panel of Figure 1 shows that autocorrelations of daily squared returns are significant up to more than a six-month lag (126 trading days). The bottom panel shows that correlations between daily returns and future squared returns are negative and significant up to a two-month lag (42 trading days). We use these critical empirical facts as the basis for our benchmark estimation. We then consider 
the moments

$$
\left\{E\left[r_{t}^{2} r_{t+j}^{2}\right]\right\}_{j=1 \text { to } 126} \text { and }\left\{E\left[r_{t} r_{t+j}^{2}\right]\right\}_{j=1 \text { to } 42}
$$

The return series has a standard deviation of 8.39E-3, a skewness of -0.8077 and an excess kurtosis of 15.10, and these sample estimates are all significant at the $5 \%$ level. We then add the moments

$$
\left\{E\left[r_{t}^{n}\right]\right\}_{n=2 \text { to } 4}
$$

in order to match this significant variance, skewness and kurtosis. Thus, in total, our benchmark estimation uses $126+42+3=171$ moments and the corresponding results are provided in Panel A of Table 1.

Starting with the SVS model, Panel A of Table 1 shows that $\beta_{1}$ is negative for the one-factor SVS and both $\beta_{1}$ and $\beta_{2}$ are negative for the two-factor SVS. These coefficients are all significant at conventional levels, as well as all the coefficients describing the factor dynamics. The SVS model thus generates the well-documented negative leverage effect. Contemporaneous asymmetry does not seem to be important for the historical distribution of returns. For the one-factor SVS model, the minimum distance between actual and model-implied moments is 46.23 when $\eta_{1}$ is estimated, and 46.77 when $\eta_{1}$ is constrained to zero. The difference of 0.54 that follows a $\chi^{2}(1)$ is not statistically significant, since its $p$-value of 0.46 is larger than conventional levels.

The minimum distance between actual and model-implied moments is 32.27 for the SVS2FU model. The difference from the SVS1FC model is then 14.50 and follows a $\chi^{2}(6)$. It appears to be statistically significant, since the associated $p$-value is 0.02 , showing that the SVS2FU model outperforms the one-factor SVS model. The SVS2FU model has a long-run volatility component with a persistence of 0.99148 , a half-life of 81 days, as well as a short-run volatility component with a persistence of 0.81028 , a half-life of approximately three days. The factor persistence in the one-factor SVS model, 0.98235 for the SVS1FU and 0.97985 for the SVS1FC, is intermediate between these long- and short-run volatility components, having a half-life of 39 days and 34 days, respectively.

Panel A of Table 1 also shows results for the GARCH models. All parameters are statistically significant at conventional levels and the parameter $\eta_{1 h}$ is negative by our new estimation strategy, 
corroborating the findings of Christoffersen et al. (2006). In addition, the "LR-test" largely rejects $\mathrm{HN}$ against both $\mathrm{CHJ}$ and CJOW, with $p$-values lower than or equal to $2 \%$, suggesting that conditional skewness as well as more than one factor are both important features of the historical returns distribution. It is important to note that the long- and short-run volatility components implied by CJOW have persistence, 0.99193 and 0.80466, comparable to those of their analogue implied by the SVS2FU model, 0.99148 and 0.81028 respectively. The volatility persistence in CHJ and HN, 0.98232 and 0.98457 respectively, is also intermediate between the long- and short-run volatility components.

Although the SVS1FC model and HN have the same number of parameters, the fit of actual moments is different. The fit is better for the SVS1FC model, 46.77, compared to 52.94 for HN, a substantial difference of 6.17 , attributable to conditional skewness in the SVS1FC model. Also note that the fit of the SVS1FC model and CHJ is comparable, 46.77 against 46.35, although the SVS1FC has one less parameter. Non-reported results show that several constrained versions of the two-factor SVS model cannot be rejected against the SVS2FU model, and they all outperform CJOW as well. We examine one of these constrained versions in more detail in the option-pricing empirical analysis.

To further visualize how well the models reproduce the stylized facts, we complement the results in Panel A of Table 1 by plotting the model-implied autocorrelations and cross-correlations together with actual ones in Figure 2, for both SVS and GARCH. The figure highlights the importance of a second factor in matching autocorrelations and cross-correlations at both the short and the long horizons. In particular, a second factor is necessary to match long-horizon autocorrelations and cross-correlations.

Panel B of Table 1 shows the estimation results when we decide to match the correlations between returns and future squared returns up to only 21 days instead of the 42 days in Panel A. In Panel B, we therefore eliminate 21 moments from the estimation. All the findings in Panel A still hold in Panel B. In the external appendix, a table shows the estimation results over the subsample starting January 2, 1981. All findings reported for the full sample are confirmed over this subsample. 


\section{SVS vs. GARCH Models: Option-Pricing Analysis}

\subsection{Option Pricing with Stochastic Skewness}

In this section, we assume that both GARCH and SVS dynamics are under the risk-neutral measure. Hence we have

$$
E\left[\exp \left(r_{t+1}\right) \mid I_{t}\right]=\exp \left(r_{f}\right)
$$

where $r_{t+1}$ and $r_{f}$ refer to the risky return and the constant risk-free rate from date $t$ to date $t+1$, respectively. In particular, for the SVS model, the pricing restriction (39) implies that the coefficients $\lambda_{0}$ and $\lambda_{i}, i=1, \ldots, K$ are given by

$$
\begin{aligned}
& \lambda_{0}=r_{f}+\sum_{i=1}^{K} \nu_{i}\left(\beta_{i} \alpha_{i}+\ln \left(1-\alpha_{i}\left(\beta_{i}+\psi\left(1 ; \eta_{i}\right)\right)\right)\right), \\
& \lambda_{i}=\phi_{i}\left(\beta_{i}-\frac{\beta_{i}+\psi\left(1 ; \eta_{i}\right)}{1-\alpha\left(\beta_{i}+\psi\left(1 ; \eta_{i}\right)\right)}\right), \quad i=1, \ldots, K .
\end{aligned}
$$

Because all models considered in this paper are affine, the price at date $t$ of a European call option with strike price $X$ and maturity $\tau$ admits a closed-form formula, reported in the external appendix owing to space limitations. We next discuss the option data used in our empirical analysis. Then we estimate the models by maximizing the fit to our option data.

\subsection{Option Data}

We use closing prices on European S\&P500 index options from OptionMetrics for the period January 1, 1996 through December 31, 2004. In order to ensure that the contracts we use are liquid, we rely on only options with maturity between 15 and 180 days. For each maturity on each Wednesday, we retain only the seven most liquid strike prices. We restrict attention to Wednesday data to enable us to study a fairly long time-period while keeping the size of the data set manageable. Our sample has 10,138 options. Using Wednesday is common practice in the literature, to limit the impact of holidays and day-of-the week effects (see Heston and Nandi 2000; Christoffersen and Jacobs 2004).

Table 2 describes key features of the data. The top panel of Table 2 sorts the data by six mon- 
eyness categories and reports the number of contracts, the average option price, the average BlackScholes implied volatility (IV), and the average bid-ask spread in dollars. Moneyness is defined as the implied index futures price, $F$, divided by the option strike price $X$. The implied-volatility row shows that deep out-of-the-money puts, those with $F / X>1.06$, are relatively expensive. The implied-volatility for those options is $25.73 \%$, compared with $19.50 \%$ for at-the-money options. The data thus display the well-known smirk pattern across moneyness. The middle panel sorts the data by maturity reported in calendar days. The IV row shows that the term structure of volatility is roughly flat, on average, during the period, ranging from $20.69 \%$ to $21.87 \%$. The bottom panel sorts the data by the volatility index (VIX) level. Obviously, option prices and IVs are increasing in VIX, and dollar spreads are increasing in VIX as well. More importantly, most of our data are from days with VIX levels between $15 \%$ and $35 \%$.

\subsection{Estimating Model Parameters from Option Prices}

As is standard in the derivatives literature, we next compare the option-pricing performance of HN, CHJ, CJOW, SVS1FU, SVS1FC, SVS2FU and the 8-parameter two-factor model with the constraints $\eta_{1}=0$ and $\beta_{2}=0$, which we further denote as SVS2FC. We use the implied-volatility root mean squared error (IVRMSE) to measure performance. Renault (1997) discusses the benefits of using the IVRMSE metric for comparing option-pricing models. To obtain the IVRMSE, we invert each computed model option price $C_{j}^{M o d}$ using the Black-Scholes formula, to get the implied volatility $I V_{j}^{M o d}$. We compare these model IVs to the market IV from the option data set, denoted $I V_{j}^{\text {Market }}$, which is also computed by inverting the Black-Scholes formula. The IVRMSE is now computed as

$$
I V R M S E \equiv \sqrt{\frac{1}{N} \sum_{j=1}^{N} e_{j}^{2}}
$$

where $e_{j} \equiv I V_{j}^{M k t}-I V_{j}^{M o d}$ and where $N$ denotes the total number of options in the sample. We estimate the risk-neutral parameters by maximizing the Gaussian IV option-error likelihood:

$$
\ln L^{O} \propto-\frac{1}{2} \sum_{j=1}^{N}\left(\ln \left(I V R M S E^{2}\right)+e_{j}^{2} / I V R M S E^{2}\right) .
$$


Model option prices $C_{j}^{M o d}$ depend on time-varying factors. In the GARCH option-pricing literature, it is standard to compute the volatility process using the GARCH volatility recursion, since the factors are observable. Factors in the SVS models, however, are latent, and we need to filter them in order to price options. To remain consistent and facilitate comparison with the GARCH alternative, we develop a simple GARCH recursion that approximates the volatility dynamics in the SVS model by matching the mean, variance, persistence and covariance with the returns of each volatility component. The dynamics of each volatility component is then approximated using Heston and Nandi's (2000) GARCH recursion (3), where the GARCH coefficients are expressed in terms of the associated SVS factor coefficients, as follows:

$$
\begin{aligned}
& \mu_{i h}=\mu_{i}+\left(1-\phi_{i}^{2}\right) \omega_{i} \beta_{i}^{2} \text { and } \phi_{i h}=\phi_{i}, \\
& \alpha_{i h}=\phi_{i} \sqrt{\frac{\mu_{i} \omega_{i}\left(1-\phi_{i}^{2}\right)}{2 \mu_{i h}}}\left(1+\frac{2\left(1-\phi_{i}\right) \omega_{i} \beta_{i}^{2}}{\mu_{i}}\right) \text { and } \beta_{i h}=-\beta_{i} \sqrt{\frac{\left(1-\phi_{i}^{2}\right) \omega_{i}}{2 \mu_{i} \mu_{i h}}} .
\end{aligned}
$$

Our matching procedure can be viewed as a second-order GARCH approximation of the SVS dynamics, intuitively analogue to the approximation of the log characteristic function used by Bates (2006) when filtering affine latent processes.

The top panel of Table 3 reports the results of the option-based estimation for SVS models, and the bottom panel reports the results of the GARCH alternative. All parameters are significantly estimated at the $1 \%$ level. Compared to historical parameters, the risk-neutral dynamics is more negatively skewed and the variance components are more persistent. These two findings are very common in the option-pricing literature. Higher negative skewness of the risk-neutral dynamics is reflected in higher negative values of $\beta_{i}$ and $\eta_{i}$ estimates for SVS models, and a larger negative value of $\beta_{i h}$ estimates for GARCH models. For example, the estimated values of $\beta_{1}$ and $\eta_{1}$ for the SVS1FU model are, respectively, -2450 and -0.2325 for the risk-neutral dynamics in Table 3, compared to -500 and -0.00364 respectively for the historical dynamics in Panel A of Table 1. The persistence of the variance for the SVS1FU model is 0.9920 for the risk-neutral dynamics in Table 3 and 0.9824 for the historical dynamics in Panel A of Table 1. The risk-neutral variance is more persistent than the physical variance. Also, note that, for the SVS2FU model, both volatility components are now very persistent under the risk-neutral dynamics, with half-lives of 30 days for 
the short-run component and 385 days for the long-run component, compared to 3 days and 81 days, respectively, under the historical dynamics.

The last three rows of each panel in Table 3 show the log likelihood, the IVRMSE metric of the models and their ratios relative to HN. The IVRMSE for the restricted one-factor SVS model, SVS1FC, outperforms its one-factor GARCH competitors, HN and CHJ. The IVRMSE for the SVS1FC model is $3.56 \%$, compared with $3.89 \%$ and $3.78 \%$ for $\mathrm{HN}$ and CHJ, corresponding to an improvement of $9.38 \%$ and $6.35 \%$, respectively. Moving to the unrestricted one-factor SVS model, SVS1FU, considerably reduces the pricing error and yields an impressive improvement of $23.26 \%$ and $19.85 \%$ over $\mathrm{HN}$ and CHJ, respectively. This result illustrates the superiority of our conditional skewness modeling approach over existing affine GARCH, since CHJ has the same number of parameters as the SVS1FU model, and more than the SVS1FC model. This result also highlights the clear benefit of allowing more negative skewness in the risk-neutral conditional distribution of returns.

Not surprisingly, the two-factor GARCH model (i.e., CJOW), with a RMSE of $3.00 \%$, fits the option data better than the one-factor GARCH and SVS models combined. In fact, as pointed out by Christoffersen et al. (2008) and Christoffersen et al. (2009), a second volatility factor is needed to fit appropriately the term structure of risk-neutral conditional moments. Our restricted two-factor SVS model, SVS2FC, has a comparable fit to CJOW, with a RMSE of $2.98 \%$. The performance of the unconstrained two-factor SVS model is almost similar to the constrained version, reflecting the fact that both $\eta_{1}$ and $\beta_{2}$ are not significantly estimated at the conventional $5 \%$ level. Option pricing thus seems to favor a risk-neutral distribution of stock prices that features a Gaussian as well as a negatively skewed shock; i.e., a discrete-time counterpart to a continuous-time jump-diffusion model.

Overall, the results of model estimation based on option data confirm the main conclusions from the GMM estimation based on returns in Section 4.3. Both conditional skewness in returns and a second volatility factor are necessary to reproduce the observed stylized facts, and disentangling the dynamics of conditional volatility from the dynamics of conditional skewness offers substantial improvement in fitting the distribution of asset prices.

In Table 4, we dissect the overall IVRMSE results reported in Table 3 by sorting the data by 
moneyness, maturity and VIX levels, using the bins from Table 2. The top panel of Table 4 reports the IVRMSE for the seven models by moneyness. We see that the two-factor models, with the lowest overall IVRMSE in Table 3, also have the lowest IVRMSE in each of the six moneyness categories. The benefits offered by the two-factor models are therefore not restricted to any particular subset of strike prices. Note that all models tend to perform worst for deep out-of-the-money put options $(F / X>1.06)$, also corresponding to the highest average implied volatility, as shown by the top panel of Table 2. For example, HN, CHJ and the SVS1FC model fit deep OTM options with IVRMSEs of $5.54 \%, 5.25 \%$ and $5.04 \%$, respectively. With a fit of $4.02 \%$, CJOW is outperformed by the SVS1FU, the SVS2FC and the SVS2FU models, which have fits of $3.81 \%, 3.60 \%$ and $3.58 \%$, respectively.

For deep-in-the-money options $(F / X<0.96)$, CJOW has the best fit, an IVRMSE of $2.99 \%$, compared to more than $3.31 \%$ for any other concurrent model. For all other moneyness categories, CJOW and the two-factor SVS models have comparable fits, the difference in their IVRMSEs being less than $0.10 \%$. The performance of the SVS1FU model is very consistent across strikes as well, the best among the one-factor models. Thus, contrary to the historical dynamics of returns, contemporaneous asymmetry appears to be important in characterizing the risk-neutral dynamics, and the low IVRMSE of the SVS1FU model compared to the SVS1FC model across all categories demonstrates the impact of the significant and negative estimate of $\eta_{1}$ reported in Table 3 .

The middle panel of Table 4 reports the IVRMSE across maturity categories. Again we see that all two-factor models have a comparable fit across all maturity categories, but the shortest maturity $(D T M<30)$ where the two-factor SVS models perform better compared to CJOW. The SVS1FU model still outperforms any other one-factor competitor along the maturity dimension. While all models have relatively more difficulty fitting the shortest and the longest maturity compared to intermediate maturity options, our results show the superiority of the SVS over the GARCH regarding the shortest maturity options. With an IVRMSE of $3.14 \%$ for this category, the SVS1FU model provides the best SVS fit, while the best GARCH fit of $3.66 \%$ is due to CJOW.

The bottom panel of Table 4 reports the IVRMSE across VIX levels. The two-factor models provide the best fit across the six VIX categories, and the SVS1FU model remains the best onefactor fit along this dimension. All models have difficulty fitting options when the level of market 
volatility is high (VIX $\geq 35 \%$ ), since, for all models, the IVRMSE is the highest for these VIX levels.

To summarize, our option results show that, focusing on single-factor models, the empirical performance of the SVS is superior to that of affine GARCH models in fitting the risk-neutral distribution of asset prices inferred from option data. However, while the two-factor affine GARCH model is comparable to the two-factor SVS in the overall fit, results across different option categories are mixed.

\section{Conclusion and Future Work}

This paper presents a new approach for modeling conditional skewness in a discrete-time affine stochastic volatility model. The model explicitly allows returns to be asymmetric conditional on factors and past information. This contemporaneous asymmetry is shown to be particularly important for the model to fit option data. An empirical investigation suggests that the flexibility that the model offers for conditional skewness increases its option-pricing performance relative to existing affine GARCH models. In particular, the one-factor SVS model with contemporaneous asymmetry outperforms existing one-factor affine GARCH models across all option categories.

The paper also develops a methodology for estimating the historical distribution parameters of a multivariate latent-factor model that nests the proposed SVS as well as existing affine GARCH models. The setting allows for model comparison along the same statistics, the minimum distance between model-implied and actual sample return moments.

In future research, it would be interesting to study the implications of the model for a parsimonious multiple-returns setting as well. An interesting exercise would be to look at "skewness transmission" among international stock markets. 


\section{References}

Andersen, T. G., Chung, H. J., and Sorensen, B. E. (1999), "Efficient Method of Moments Estimation of a Stochastic Volatility Model: A Monte Carlo Study," Journal of Econometrics, $91,61-87$.

Barndorff-Nielsen, O. E., and Shephard, N. (2001), "Non-Gaussian Ornstein-Uhlenbeck-Based Models and Some of Their Uses in Financial Economics," Journal Of The Royal Statistical Society Series B, 63 (2), 167-241.

Bates, D. S. (2006), "Maximum Likelihood Estimation of Latent Affine Processes," Review of Financial Studies, 19 (3), 909-965.

Black, F. (1976), "Studies of Stock Price Volatility Changes," in Proceedings of the 1976 Meetings of the American Statistical Association, Business and Economic Statistics Section, pp. 177-181.

Christie, A. A. (1982), "The Stochastic Behavior of Common Stock Variances: Value, Leverage and Interest Rate Effects," Journal of Financial Economics, 10 (4), 407-432.

Christoffersen, P., and Jacobs, K. (2004), "Which GARCH Model for Option Valuation?," Management Science, 50 (9), 1204-1221.

Christoffersen, P., Heston, S., and Jacobs, K. (2006), "Option Valuation With Conditional Skewness," Journal of Econometrics, 131 (1-2), 253-284.

_ (2009), "The Shape and Term Structure of the Index Option Smirk: Why Multifactor Stochastic Volatility Models Work So Well," Management Science, 55 (12), 1914-1932.

Christoffersen, P., Jacobs, K., Ornthanalai, C., and Wang, Y. (2008), "Option Valuation With Long-Run and Short-Run Volatility Components," Journal of Financial Economics, 90 (3), 272297.

Constantine, G. M., and Savits, T. H. (1996), "A Multivariate Faà di Bruno Formula with Applications," Transactions of the American Mathematical Society, 348 (2), 503-520. 
Danielsson, J. (1994), "Stochastic Volatility in Asset Prices Estimation with Simulated Maximum Likelihood," Journal of Econometrics, 64 (1-2), 375-400.

Darolles, S., Gourieroux, C., and Jasiak, J. (2006), "Structural Laplace Transform and Compound Autoregressive Models," Journal of Time Series Analysis, 27 (4), 477-503.

Feunou, B., Jahan-Parvar, M. R., and Tédongap, R. (2011), "Modeling Market Downside Volatility," Review of Finance, in press.

Forsberg, L., and Bollerslev, T. (2002), "Bridging the Gap Between the Distribution of Realized (ECU) Volatility and ARCH Modelling (of the Euro): the GARCH-NIG Model," Journal of Applied Econometrics, 17 (5), 535-548.

Gourieroux, C., and Jasiak, J. (2006), "Autoregressive Gamma Processes," Journal of Forecasting, $25(2), 129-152$.

Harvey, C. R., and Siddique, A. (1999), "Autoregressive Conditional Skewness," Journal of Financial and Quantitative Analysis, 34 (04), 465-487.

Heston, S. (1993), "A Closed-Form Solution for Options with Stochastic Volatility with Applications to Bond and Currency Options," Review of Financial Studies, 6 (2), 327-343.

Heston, S., and Nandi, S. (2000), "A Closed-Form GARCH Option Valuation Model," Review of Financial Studies, 13 (3), 585-625.

Jacquier, E., Polson, N. G., and Rossi, P. E. (1994), "Bayesian Analysis of Stochastic Volatility Models," Journal of Business 85 Economic Statistics, 12 (4), 371-389.

Jensen, M. B., and Lunde, A. (2001), "The NIG-S\&ARCH Model: A Fat-Tailed, Stochastic, and Autoregressive Conditional Heteroskedastic Volatility Model," Econometrics Journal, 4 (2), 319342.

Jiang, G. J., and Knight, J. L. (2002), "Estimation of Continuous-Time Processes via the Empirical Characteristic Function," Journal of Business \& Economic Statistics, 20 (2), 198-212. 
Le, A., Singleton, K. J., and Dai, Q. (2010), "Discrete-Time Affine Term Structure Models with Generalized Market Prices of Risk," Review of Financial Studies, 23 (5), 2184-2227.

Liesenfeld, R., and Jung, R. C. (2000), "Stochastic Volatility Models: Conditional Normality versus Heavy-Tailed Distributions," Journal of Applied Econometrics, 15 (2), 137-160.

Magnus, J., and Neudecker, H. (1988) Matrix Differential Calculus with Applications in Statistics and Econometrics, Chichester, U.K: Wiley Series in Probability and Statistics.

Renault, E. (1997) "Econometric Models of Option Pricing Errors," in Advances in Economics and Econometrics: Theory and Applications, Seventh World Congress, eds. D.M. Kreps and K.F. Wallis, Cambridge: Cambridge University Press, pp. 223-278.

Singleton, K. J. (2006) Empirical Dynamic Asset Pricing: Model Specification and Econometric Assessment, Princeton, NJ: Princeton University Press. 
Autocorrelations of Squared Returns

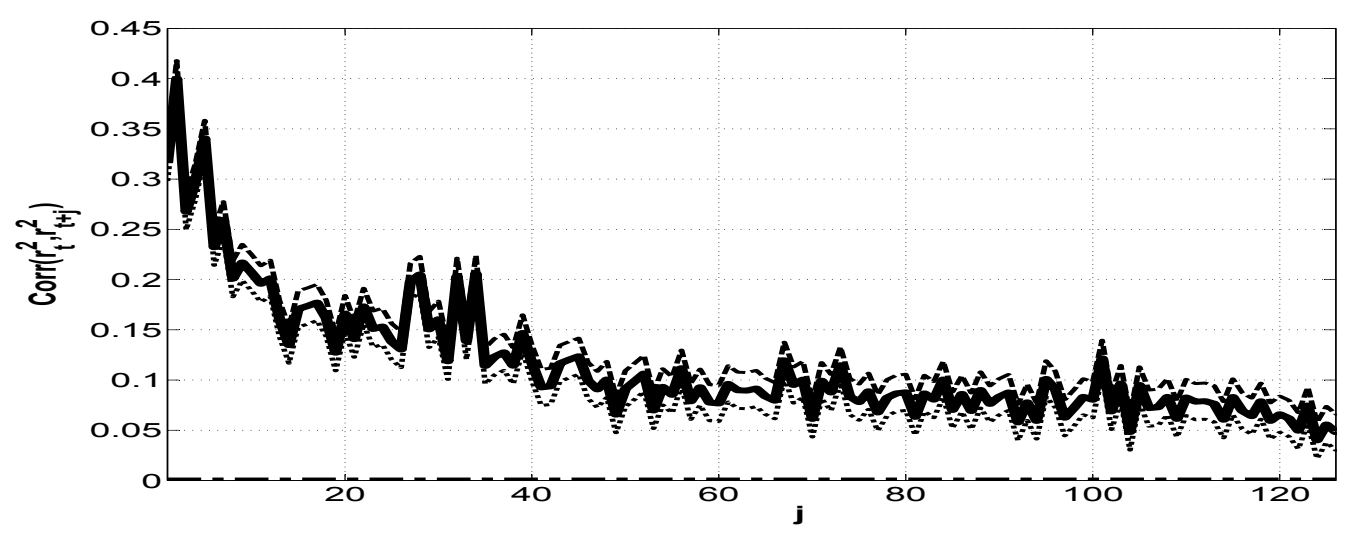

Cross-Correlations between Returns and Squared Returns

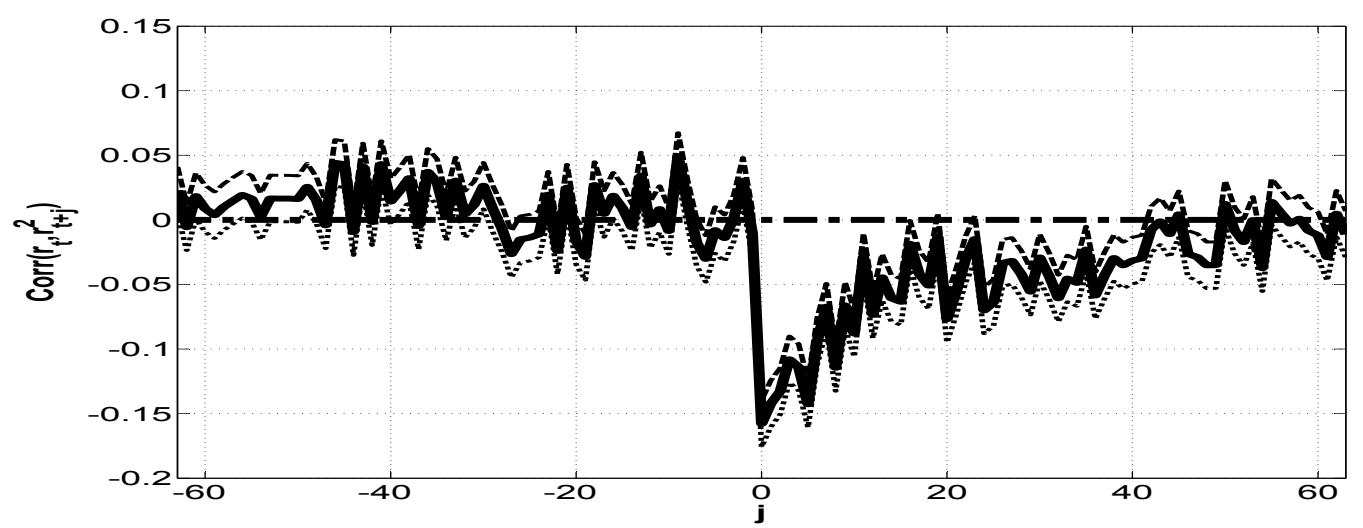

The figure shows the actual autocorrelations of squared returns, $\operatorname{Corr}\left(r_{t}^{2}, r_{t+j}^{2}\right)$, in the top graph, and the actual cross-correlations between returns and squared returns, $\operatorname{Corr}\left(r_{t}, r_{t+j}^{2}\right)$, in the bottom graph, for the S\&P500 equalweighted index. The data are sampled at the daily frequency and cover the period from January 2, 1962 to December 31,2010 , for a total of 12,336 observations. The dashed and the dotted line represent the $95 \%$ confidence bounds of the correlation.

Figure 1: Returns and Squared Returns: Stylized Facts 
SVS

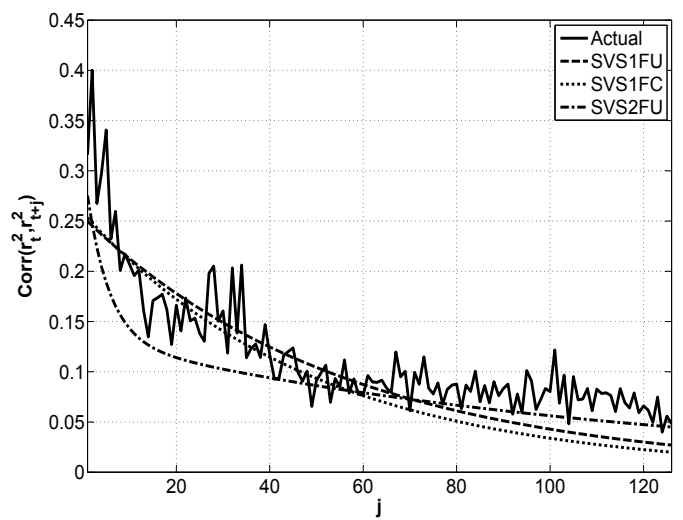

GARCH

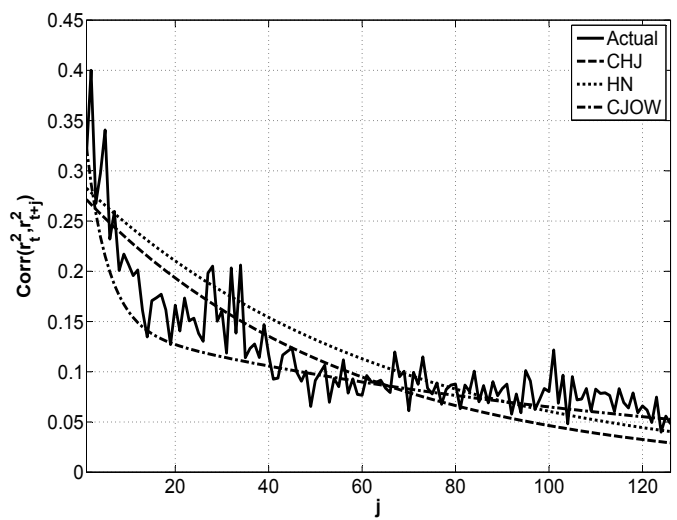

Cross-Correlations between Returns and Squared Returns

\section{SVS}

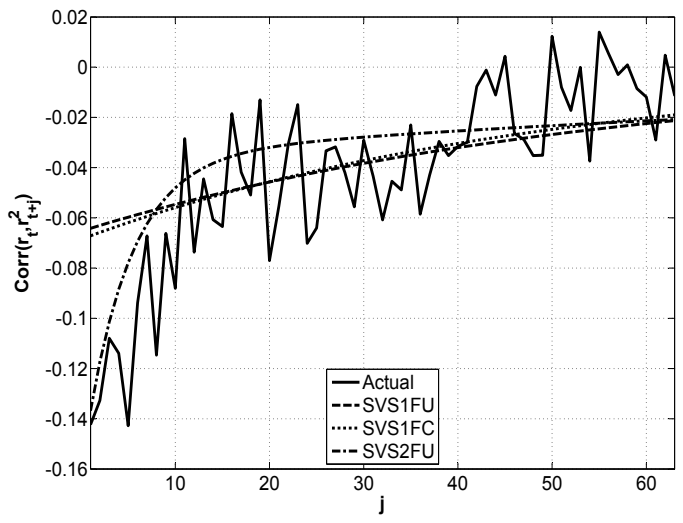

GARCH

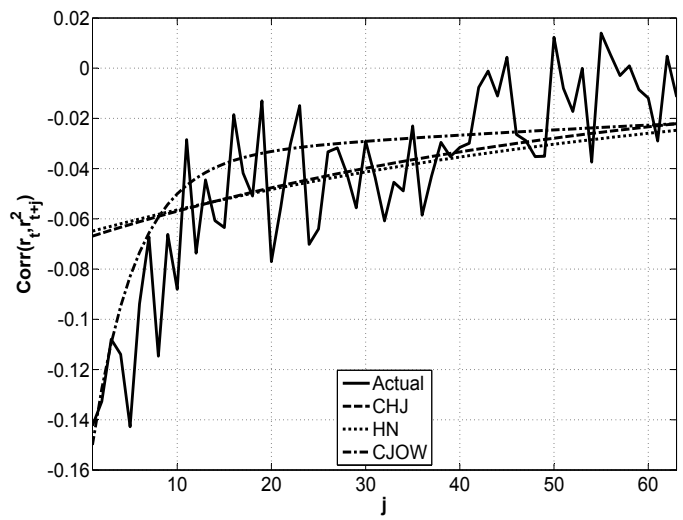

The figure shows the model-implied autocorrelations of squared returns, $\operatorname{Corr}\left(r_{t}^{2}, r_{t+j}^{2}\right)$, for SVS dynamics in the top left graph and for GARCH dynamics in the top right graph. It also shows the model-implied cross-correlation between returns and squared returns, $\operatorname{Corr}\left(r_{t}, r_{t+j}^{2}\right)$, for SVS dynamics in the bottom left graph and for GARCH dynamics in the bottom right. Returns are those of the S\&P500 equal-weighted index. The data are sampled at the daily frequency and cover the period from January 2, 1962 to December 31, 2010, for a total of 12,336 observations.

\section{Figure 2: Returns and Squared Returns: Model Implications}




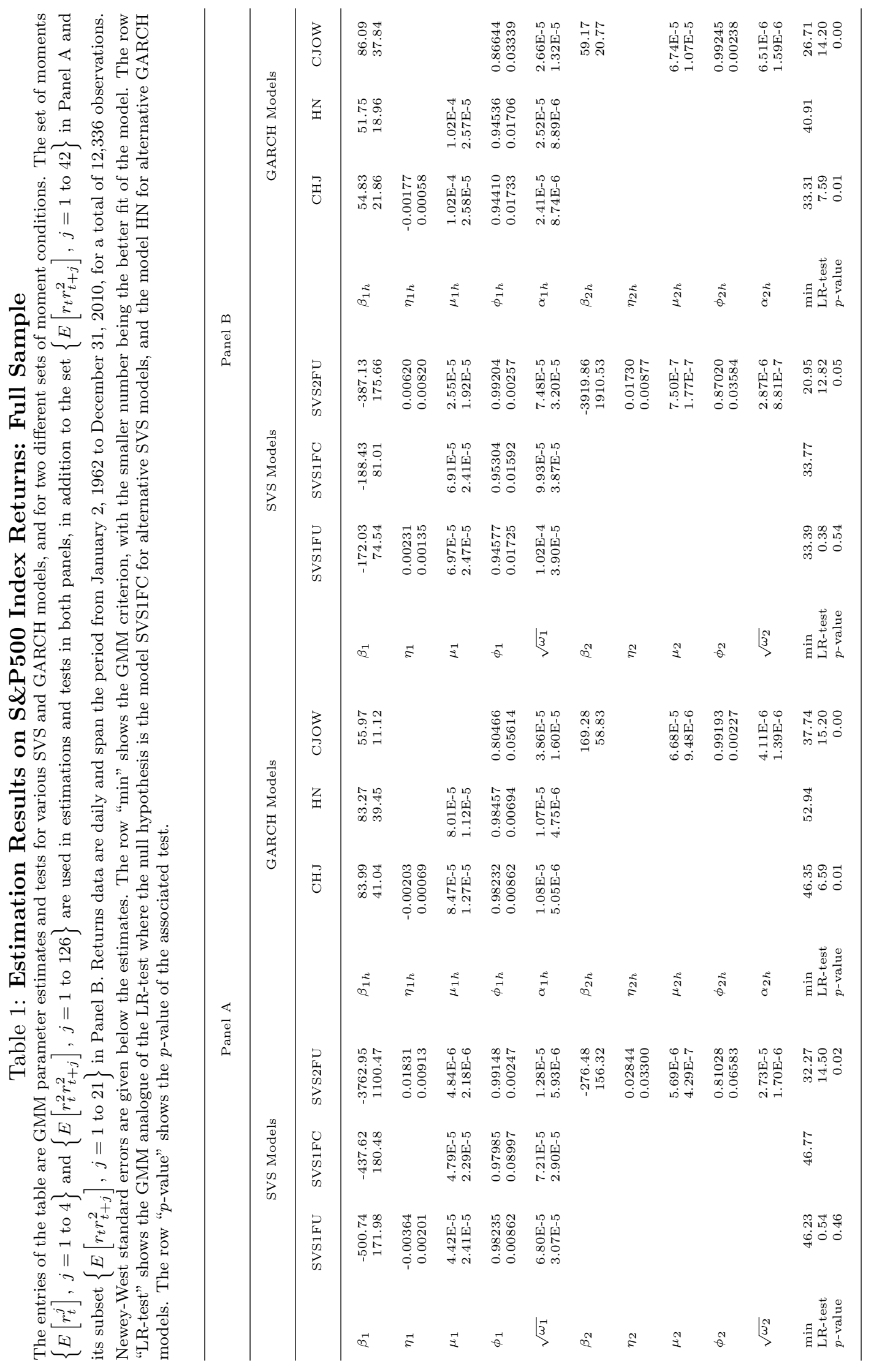




\section{Table 2: S\&P500 Index Option Data}

We use Wednesday closing out-of-the-money (OTM) call and put option data from OptionMetrics from January 1, 1996 through December 31, 2004. $F$ denotes the implied futures price of the S\&P500 index, $X$ denotes the strike price and DTM denotes the number of calendar days to maturity. The average bid-ask spread is reported in dollars.

\begin{tabular}{|c|c|c|c|c|c|c|c|}
\hline Moneyness $F / X$ & $0.96-$ & $0.96+, 0.98-$ & $0.98+, 1.02-$ & $1.02+, 1.04-$ & $1.04+, 1.06-$ & $1.06+$ & All \\
\hline Nb Contracts & 1,162 & 961 & 3,294 & 1,325 & 951 & 2,445 & 10,138 \\
\hline Avg Price & 17.41 & 22.37 & 30.59 & 25.55 & 22.13 & 17.51 & 23.69 \\
\hline Avg Implied Vol & 19.63 & 18.71 & 19.50 & 21.23 & 22.22 & 25.73 & 21.42 \\
\hline Avg Bid-Ask Spread & 1.187 & 1.378 & 1.572 & 1.400 & 1.298 & 1.154 & 1.361 \\
\hline DTM & $30-$ & $30+, 60-$ & $60+, 90-$ & $90+, 120-$ & $120+, 150-$ & $150+$ & All \\
\hline $\mathrm{Nb}$ Contracts & 695 & 3,476 & 2,551 & 1,063 & 1,332 & 1,021 & 10,138 \\
\hline Avg Price & 12.11 & 17.97 & 24.47 & 27.51 & 31.20 & 35.36 & 23.69 \\
\hline Avg Implied Vol & 20.69 & 21.04 & 21.62 & 21.86 & 21.75 & 21.87 & 21.42 \\
\hline Avg Bid-Ask Spread & 0.830 & 1.184 & 1.452 & 1.539 & 1.588 & 1.610 & 1.361 \\
\hline VIX Level & $15-$ & $15+, 20-$ & $20+, 25-$ & $25+, 30-$ & $30+, 35-$ & $35+$ & All \\
\hline $\mathrm{Nb}$ Contracts & 625 & 2,844 & 3,873 & 1,621 & 781 & 394 & 10,138 \\
\hline Avg Price & 14.10 & 16.49 & 25.22 & 30.28 & 29.61 & 37.10 & 23.69 \\
\hline Avg Implied Vol & 13.83 & 17.35 & 21.38 & 25.26 & 28.76 & 33.06 & 21.42 \\
\hline Avg Bid-Ask Spread & 0.958 & 1.014 & 1.397 & 1.694 & 1.743 & 2.007 & 1.361 \\
\hline
\end{tabular}


Table 3: Estimation Results on S\&P500 Index Options: SVS and GARCH Models We estimate the four SVS and the three GARCH models using option data for the period January 1, 1996 to December 31, 2004. Standard errors, computed using the outer product of the gradient, are indicated in parentheses.

\begin{tabular}{|c|c|c|c|c|c|c|c|c|}
\hline \multirow[b]{3}{*}{ Parameters } & \multicolumn{6}{|c|}{ SVS Models } & & \\
\hline & \multicolumn{2}{|c|}{ SVS1FC } & \multicolumn{2}{|c|}{ SVS1FU } & \multicolumn{2}{|c|}{ SVS2FC } & \multicolumn{2}{|c|}{ SVS2FU } \\
\hline & Estimate & Std Error & Estimate & Std Error & Estimate & Std Error & Estimate & Std Error \\
\hline$\beta_{1}$ & $-2.468 \mathrm{E}+3$ & $(1.28 \mathrm{E}+2)$ & $-2.450 \mathrm{E}+3$ & $(8.02 \mathrm{E}+1)$ & $-1.046 \mathrm{E}+4$ & $(1.15 \mathrm{E}+3)$ & $-1.847 \mathrm{E}+4$ & $(2.80 \mathrm{E}+3)$ \\
\hline$\eta_{1}$ & $0.000 \mathrm{E}+0$ & & $-2.325 \mathrm{E}-1$ & $(1.08 \mathrm{E}-2)$ & $0.000 \mathrm{E}+0$ & & $1.083 \mathrm{E}-2$ & $(3.92 \mathrm{E}-2)$ \\
\hline$\mu_{1}$ & $3.968 \mathrm{E}-5$ & $(1.55 \mathrm{E}-6)$ & $8.534 \mathrm{E}-5$ & $(1.58 \mathrm{E}-6)$ & $5.566 \mathrm{E}-6$ & $(6.22 \mathrm{E}-7)$ & $3.421 \mathrm{E}-6$ & (5.13E-7) \\
\hline$\phi_{1}$ & $9.879 \mathrm{E}-1$ & $(2.13 \mathrm{E}-4)$ & $9.920 \mathrm{E}-1$ & $(1.59 \mathrm{E}-4)$ & $9.763 \mathrm{E}-1$ & $(5.83 \mathrm{E}-4)$ & $9.770 \mathrm{E}-1$ & $(7.75 \mathrm{E}-4)$ \\
\hline$\sqrt{\omega_{1}}$ & $2.315 \mathrm{E}-5$ & $(8.98 \mathrm{E}-7)$ & $2.684 \mathrm{E}-5$ & $(4.96 \mathrm{E}-7)$ & $3.616 \mathrm{E}-6$ & $(3.88 \mathrm{E}-7)$ & $2.097 \mathrm{E}-6$ & $(3.08 \mathrm{E}-7)$ \\
\hline$\beta_{2}$ & & & & & $0.000 \mathrm{E}+0$ & & $-4.025 \mathrm{E}-1$ & $(7.51 \mathrm{E}+1)$ \\
\hline$\eta_{2}$ & & & & & $-1.975 \mathrm{E}-1$ & $(5.88 \mathrm{E}-3)$ & $-2.018 \mathrm{E}-1$ & $(5.82 \mathrm{E}-3)$ \\
\hline$\mu_{2}$ & & & & & $2.592 \mathrm{E}-5$ & $(7.71 \mathrm{E}-6)$ & $5.275 \mathrm{E}-5$ & $(3.39 \mathrm{E}-6)$ \\
\hline$\phi_{2}$ & & & & & $9.989 \mathrm{E}-1$ & $(1.60 \mathrm{E}-4)$ & $9.982 \mathrm{E}-1$ & $(1.35 \mathrm{E}-4)$ \\
\hline$\sqrt{\omega_{2}}$ & & & & & $6.672 \mathrm{E}-5$ & $(4.42 \mathrm{E}-6)$ & $5.275 \mathrm{E}-5$ & $(1.49 \mathrm{E}-6)$ \\
\hline
\end{tabular}

\section{Model Properties}

\begin{tabular}{|c|c|c|c|c|}
\hline Log Likelihood & 20,100 & 21,080 & 21,851 & 21,880 \\
\hline IVRMSE & 3.557 & 3.156 & 2.981 & 2.970 \\
\hline Ratio to $\mathrm{HN}$ & 0.914 & 0.811 & 0.766 & 0.763 \\
\hline
\end{tabular}

GARCH Models

\begin{tabular}{|c|c|c|c|c|c|c|}
\hline \multirow[b]{2}{*}{ Parameters } & \multicolumn{2}{|c|}{$\mathbf{H N}$} & \multicolumn{2}{|c|}{ CHJ } & \multicolumn{2}{|c|}{ CJOW } \\
\hline & Estimate & Std Error & Estimate & Std Error & Estimate & Std Error \\
\hline $\begin{array}{l}\mu_{1 h} \\
\phi_{1 h} \\
\alpha_{1 h} \\
\beta_{1 h} \\
\eta_{1 h}\end{array}$ & $\begin{array}{r}1.24 \mathrm{E}-4 \\
9.85 \mathrm{E}-1 \\
1.81 \mathrm{E}-6 \\
208.61\end{array}$ & $\begin{array}{r}(6.55 \mathrm{E}-7) \\
(8.97 \mathrm{E}-5) \\
(5.85 \mathrm{E}-9) \\
(4.62 \mathrm{E}+0)\end{array}$ & $\begin{array}{r}1.178 \mathrm{E}-4 \\
0.9857 \\
1.68 \mathrm{E}-6 \\
238.50 \\
-2.17 \mathrm{E}-3\end{array}$ & $\begin{array}{r}(9.04 \mathrm{E}-7) \\
(1.27 \mathrm{E}-4) \\
(2.04 \mathrm{E}-8) \\
(5.84 \mathrm{E}+0) \\
(8.45 \mathrm{E}-5)\end{array}$ & $\begin{array}{r}8.01 \mathrm{E}-5 \\
9.66 \mathrm{E}-1 \\
2.44 \mathrm{E}-6 \\
291.60\end{array}$ & $\begin{array}{r}(3.34 \mathrm{E}-6) \\
(7.22 \mathrm{E}-4) \\
(1.39 \mathrm{E}-7) \\
(1.60 \mathrm{E}+1)\end{array}$ \\
\hline $\begin{array}{l}\phi_{2 h} \\
\alpha_{2 h} \\
\beta_{2 h}\end{array}$ & & & & & $\begin{array}{r}9.97 \mathrm{E}-1 \\
2.19 \mathrm{E}-6 \\
2.22 \mathrm{E}+1\end{array}$ & $\begin{array}{r}(1.49 \mathrm{E}-4) \\
(5.67 \mathrm{E}-8) \\
(2.13 \mathrm{E}+0)\end{array}$ \\
\hline \multicolumn{7}{|c|}{ Model Properties } \\
\hline $\begin{array}{l}\text { Log Likelihood } \\
\text { IVRMSE } \\
\text { Ratio to HN }\end{array}$ & \multicolumn{2}{|c|}{$\begin{array}{c}19,158 \\
3.890 \\
1.000\end{array}$} & & $\begin{array}{l}277 \\
83 \\
72\end{array}$ & & $\begin{array}{l}623 \\
700 \\
71\end{array}$ \\
\hline
\end{tabular}


Table 4: IVRMSE Option Error by Moneyness, Maturity and VIX Level

We use Wednesday closing out-of-the-money (OTM) call and put option data from OptionMetrics from January 1, 1996 through December 31, 2004. $F$ denotes the implied futures price of the S\&P500 index, $X$ denotes the strike price and DTM denotes the number of calendar days to maturity. We report IVRMSE from the models estimated in Table 3 by moneyness, maturity and VIX level.

\begin{tabular}{|c|c|c|c|c|c|c|}
\hline Moneyness $F / X$ & $0.96-$ & $0.96+, 0.98-$ & $0.98+, 1.02-$ & $1.02+, 1.04-$ & $1.04+, 1.06-$ & $1.06+$ \\
\hline $\mathrm{HN}$ & 3.432 & 3.328 & 3.275 & 3.308 & 3.588 & 5.539 \\
\hline CHJ & 3.483 & 3.207 & 3.238 & 3.253 & 3.515 & 5.251 \\
\hline CJOW & 2.993 & 2.628 & 2.557 & 2.615 & 2.746 & 4.024 \\
\hline SVS1FC & 3.314 & 3.065 & 2.923 & 3.044 & 3.253 & 5.044 \\
\hline SVS1FU & 3.609 & 2.675 & 2.720 & 2.913 & 3.096 & 3.813 \\
\hline SVS2FC & 3.658 & 2.551 & 2.492 & 2.620 & 2.802 & 3.602 \\
\hline SVS2FU & 3.633 & 2.542 & 2.491 & 2.617 & 2.800 & 3.581 \\
\hline DTM & $30-$ & $30+, 60-$ & $60+, 90-$ & $90+, 120-$ & $120+, 150-$ & $150+$ \\
\hline $\mathrm{HN}$ & 4.152 & 3.854 & 3.846 & 3.638 & 3.931 & 4.093 \\
\hline CHJ & 3.989 & 3.697 & 3.732 & 3.590 & 3.910 & 4.036 \\
\hline CJOW & 3.664 & 3.064 & 2.811 & 2.670 & 2.944 & 3.089 \\
\hline SVS1FC & 4.049 & 3.570 & 3.427 & 3.236 & 3.556 & 3.739 \\
\hline SVS1FU & 3.143 & 3.200 & 3.075 & 2.802 & 3.328 & 3.331 \\
\hline SVS2FC & 3.282 & 3.090 & 2.878 & 2.619 & 2.937 & 3.028 \\
\hline SVS2FU & 3.260 & 3.073 & 2.856 & 2.607 & 2.957 & 3.035 \\
\hline VIX Level & $15-$ & $15+, 20-$ & $20+, 25-$ & $25+, 30-$ & $30+, 35-$ & $35+$ \\
\hline $\mathrm{HN}$ & 4.489 & 3.130 & 3.280 & 4.314 & 5.199 & 7.131 \\
\hline CHJ & 4.309 & 2.887 & 3.177 & 4.188 & 5.216 & 7.300 \\
\hline CJOW & 2.855 & 2.430 & 2.814 & 3.073 & 4.085 & 5.119 \\
\hline SVS1FC & 3.482 & 2.872 & 3.400 & 4.044 & 4.537 & 4.998 \\
\hline SVS1FU & 3.251 & 2.326 & 2.877 & 3.399 & 4.497 & 5.617 \\
\hline SVS2FC & 2.897 & 2.274 & 2.683 & 3.118 & 4.391 & 5.390 \\
\hline SVS2FU & 2.963 & 2.269 & 2.655 & 3.080 & 4.344 & 5.492 \\
\hline
\end{tabular}

